\title{
Late outcomes comparison of nonelderly patients with stented bioprosthetic and mechanical valves in the aortic position: $A$ propensity-matched analysis
}

\author{
R. Scott McClure, MD, SM, ${ }^{\mathrm{a}, \mathrm{b}}$ Siobhan McGurk, BA, ${ }^{\mathrm{a}}$ Marisa Cevasco, MD, ${ }^{\mathrm{a}}$ Ann Maloney, BA, ${ }^{\mathrm{a}}$ \\ Igor Gosev, MD, ${ }^{a}$ Esther M. Wiegerinck, MD, ${ }^{a}$ Genina Salvio, PA, ${ }^{a}$ George Tokmaji, MD, ${ }^{a}$ \\ Wernard Borstlap, AB, ${ }^{a}$ Foeke Nauta, $\mathrm{AB},{ }^{a}$ and Lawrence H. Cohn, $\mathrm{MD}^{\mathrm{a}}$
}

Objective: Our study compares late mortality and valve-related morbidities between nonelderly patients (aged $<65$ years) undergoing stented bioprosthetic or mechanical valve replacement in the aortic position.

\begin{abstract}
Methods: We identified 1701 consecutive patients aged $<65$ years who underwent aortic valve replacement between 1992 and 2011. A stented bioprosthetic valve was used in 769 patients $(45 \%)$ and a mechanical valve was used in 932 patients (55\%). A stepwise logistic regression propensity score identified a subset of 361 evenly matched patient-pairs. Late outcomes of death, reoperation, major bleeding, and stroke were assessed.
\end{abstract}

\begin{abstract}
Results: Follow-up was $99 \%$ complete. The mean age in the matched cohort was 53.9 years (bioprosthetic valve) and 53.2 years (mechanical valve) $(P=.30)$. Fifteen additional measurable variables were statistically similar for the matched cohort. Thirty-day mortality was $1.9 \%$ (bioprosthetic valve) and $1.4 \%$ (mechanical valve) $(P=.77)$. Survival at $5,10,15$, and 18 years was $89 \%, 78 \%, 65 \%$, and $60 \%$ for patients with bioprosthetic valves versus $88 \%, 79 \%, 75 \%$, and $51 \%$ for patients with mechanical valves $(P=.75)$. At 18 years, freedom from reoperation was $95 \%$ for patients with mechanical valves and $55 \%$ for patients with bioprosthetic valves $(P=.002)$, whereas freedom from a major bleeding event favored patients with bioprosthetic valves $(98 \%)$ versus mechanical valves $(78 \% ; P=.002)$. There was no difference in stroke between the 2 matched groups.
\end{abstract}

Conclusions: In patients aged $<65$ years, despite an increase in the rate of reoperation with stented bioprosthetic valves and an increase in major bleeding events with mechanical valves, there is no significant difference in mortality at late follow-up. (J Thorac Cardiovasc Surg 2014;148:1931-9)

The emphasis to place on patient age with regard to valve choice in aortic stenosis remains a perplexing dilemma in select situations. The recent guidelines for aortic valve replacement have removed age as an absolute determinant in the decision-making process. Still, whether implicitly stated or not, when deciding to implant a mechanical or bioprosthetic heart valve into a patient, age will always be a consideration. The quandary between mechanical prostheses and bioprostheses endures - superior prosthesis durability and low likelihood of future reoperations with mechanical implants at the expense of lifelong anticoagulation and the associated increase in bleeding attributed to warfarin therapy. Quality of life infringements related to labor-intensive monitoring with warfarin are also not negligible.

\footnotetext{
From the Division of Cardiac Surgery, ${ }^{\text {a }}$ Harvard Medical School, Brigham and Women's Hospital, Boston, Mass; and Division of Cardiac Surgery, ${ }^{\mathrm{b}}$ Queen's University, Kingston General Hospital, Kingston, Ontario, Canada.

Disclosures: The authors have nothing to disclose with regard to commercial support.

Received for publication Sept 1, 2013; revisions received Dec 3, 2013; accepted for publication Dec 12, 2013; available ahead of print Feb 9, 2014.

Address for reprints: Lawrence H. Cohn, MD, Brigham and Women's Hospital, 75

Francis St, Boston, MA 02115 (E-mail: 1cohn@partners.org).

$0022-5223 / \$ 36.00$

Copyright (C) 2014 by The American Association for Thoracic Surgery

http://dx.doi.org/10.1016/j.jtcvs.2013.12.042
}

Prior renditions of valve guidelines recommended that patients younger than age 65 years undergo implantation of a mechanical prosthesis (barring contraindications to anticoagulation therapy) whereas patients older than age 65 years receive a bioprosthesis. ${ }^{1}$ The threshold of 65 years was considered the inflection point where the risk of reoperation secondary to structural valve deterioration (SVD) was low enough that the advantage of a bioprosthesis (ie, removal of warfarin therapy) outweighed the long-term durability inherent to a mechanical prosthesis. Improvements in surgical outcomes for reoperations, ${ }^{2,3}$ perceived improvements in the durability of newer-generation bioprostheses, ${ }^{4,5}$ and the currently untested yet highly anticipated future use of percutaneous valve-in-valve technology to treat $\mathrm{SVD}^{6}$ have contributed to a steady trend toward implanting bioprostheses into younger patients instead of mechanical prostheses. ${ }^{7,8}$

Despite this trend, there are contemporary singleinstitution observational studies that suggest a mortality benefit of mechanical prostheses over bioprosthetic prostheses in nonelderly patients. ${ }^{9-11}$ These studies suggest caution when implanting bioprostheses into patients aged younger than 65 years. In contrast, the only contemporary randomized clinical trial to assess the issue revealed no difference in survival out to 13 years. ${ }^{12}$ Considering the 


\section{Abbreviations and Acronyms \\ AVR $=$ aortic valve replacement \\ $\mathrm{EMR}=$ electronic medical record \\ SVD $=$ structural valve deterioration}

paucity of data and conflicting conclusions in the contemporary literature, we set out to compare late mortality and valve-related morbidity between nonelderly patients undergoing implantation of a stented bioprosthetic or mechanical valve in the aortic position at the Brigham and Women's Hospital. The purpose of our study was to delineate differences in outcomes at late follow-up and to see if the current trend toward implanting bioprosthetic valves into younger patient populations is justifiable.

\section{PATIENTS AND METHODS}

With approval from an institutional review board, a review of the electronic medical record (EMR) was conducted to identify all patients aged younger than 65 years undergoing an isolated aortic valve replacement (AVR) with a bileaflet mechanical or stented bioprosthesis from January 1992. An isolated AVR was defined as an AVR without additional concomitant valvular, coronary, or ventricular procedures at the time of the indexed operation. Concomitant aortic root and/or ascending aortic repair procedures were included in the isolated AVR cohort, as were patients having had cardiac surgery before the indexed operation. Exclusion criteria were AVR using a pulmonary autograft, homograft, or stentless bioprostheses. Of 6794 patients who underwent an AVR within the specified time frame, 1701 patients met the inclusion criteria. The primary outcome was late survival. Secondary outcomes included stroke, major bleeding, and reoperations at late follow-up.

The study cohort underwent a propensity-matched analysis to create evenly matched patient-pairs with respect to measurable covariates, with type of prosthesis implanted (ie, bileaflet mechanical or a secondgeneration stented bioprosthetic prosthesis) at the time of the indexed operation being the only discernible difference. The remaining unmatched patients were placed into a separate database and their outcomes were also assessed. The investigators were blinded to any outcomes during the matching process (Figure 1).

Unless warfarin therapy was indicated for other reasons (eg, atrial fibrillation or pulmonary embolism), patients receiving a bioprosthetic valve were managed solely with antiplatelet therapy (ie, daily aspirin), whereas patients receiving a mechanical valve received both antiplatelet and anticoagulation therapy in combination (ie, daily aspirin and warfarin). Goals for warfarin therapy were to maintain an international normalizing ratio between 2.0 and 3.0. The decision to implant a bioprosthetic or mechanical valve was at the discretion of the primary surgeon and the patient at the time of implantation.

\section{Data Collection}

Patient characteristics, medications, laboratory values, and in-hospital outcomes of the index surgery were collected at the time of presentation and extracted from the hospital's EMR. Data on long-term outcomes were collected by questionnaires, records requested from referring physicians, and extraction from the EMR at follow-up visits. Mortality data, including date and cause of death, were collected from the following sources: the Social Security Death Index, EMR, and the state Department of Public Health and Registry of Vital Statistics. Nineteen patients with an international residence were lost to follow-up.
To acquire up-to-date data for the secondary outcomes of stroke, major bleeding, and reoperations, questionnaires were mailed to all eligible study patients residing in the United States and presumed alive as of October $2011(\mathrm{~N}=1391)$. Questionnaires were mailed in serial succession beginning in November 2011. Two additional mailings were sent in January 2012 and March 2012 for nonresponders. Patient-reported responses were cross-referenced and corroborated with the most current EMR records on file. For those patients where a questionnaire was unattainable, the time point used for secondary outcomes was the last recorded visit on file in the EMR. A responder bias analysis was performed to assess for potential differences between patients who responded to the survey and those who elected not to participate.

Patient demographics and hospital outcomes were coded and defined according to the Society for Thoracic Surgeons Adult Cardiac Surgery database specifications, version 2.52 . In addition to late mortality and valverelated morbidities, short-term outcomes were also assessed. Short-term outcomes of interest were 30-day mortality, re-exploration for bleeding, postoperative stroke, in-hospital cardiac arrest, complete heart block, time on the ventilator, intensive care unit length of stay, and hospital length of stay. Sudden, unexplained death was considered a cardiac-related mortality.

\section{Propensity-Matched Cohort}

We conducted a matched group analysis using propensity-matched cases (stented bioprostheses) and controls (mechanical valves). Propensity to receive bioprosthetic valves was evaluated using logistic regression analyses done in 2 steps. Variables to be evaluated as predictors were selected based on literature review, known confounding covariates for the outcomes of interest, differences between the 2 patient groups (Table 1), and clinical judgment. These variables were then classified as patient-dependent or treatment-dependent and separate forward-stepwise regression analyses were conducted for each variable set, including examinations for interaction effects. Any variable with a $P \leq .15$ was entered into the final model, which was an enter-method logistic regression. The final model consisted of 11 variables: age, year of surgery, cardiopulmonary bypass time, etiology of disease (ie, calcific, endocarditis, congenital, rheumatic, primary aorta, or other), body mass index, reoperation, gender, hypertension, congestive heart failure, operative status (elective, urgent, or emergent), and ejection fraction. An interaction variable between the surgeon and the year of surgery was also included to control for differences in patient mix and clinical practice over time. The resulting adjusted predicted probability score for each patient was then used to select matched pairs based on probability scores $<.01$ (a priori algorithm).

\section{Statistical Analysis}

Normally distributed continuous variables are presented as mean \pm standard deviation. Non-normally distributed continuous variables are presented as median with interquartile range. Analyses of continuous variables were done using the Student $t$ test with Levine's homogeneity of variance or Mann-Whitney $U$ test, as appropriate. Dichotomous variables were evaluated using the Fisher exact test and are presented as a numerical value as well as a percentage. Outcomes of interest were analyzed by KaplanMeier analysis. Failure time date was compared using the log-rank test. Life-table estimations of 5-year, 10-year, 15-year, and 18-year survival curves are presented as cumulative percent \pm standard error. Statistical analyses were done using SPSS (version 13.0; IBM-SPSS Inc, Armonk, NY).

\section{RESULTS}

Our study included 1701 patients (769 stented bioprosthetic cases, 932 mechanical cases; 14,848 patient-years of data; median follow-up 8-years). Baseline characteristics for the complete cohort are presented in Table 1. Patients receiving a bioprosthesis were older and had more hypertension and 


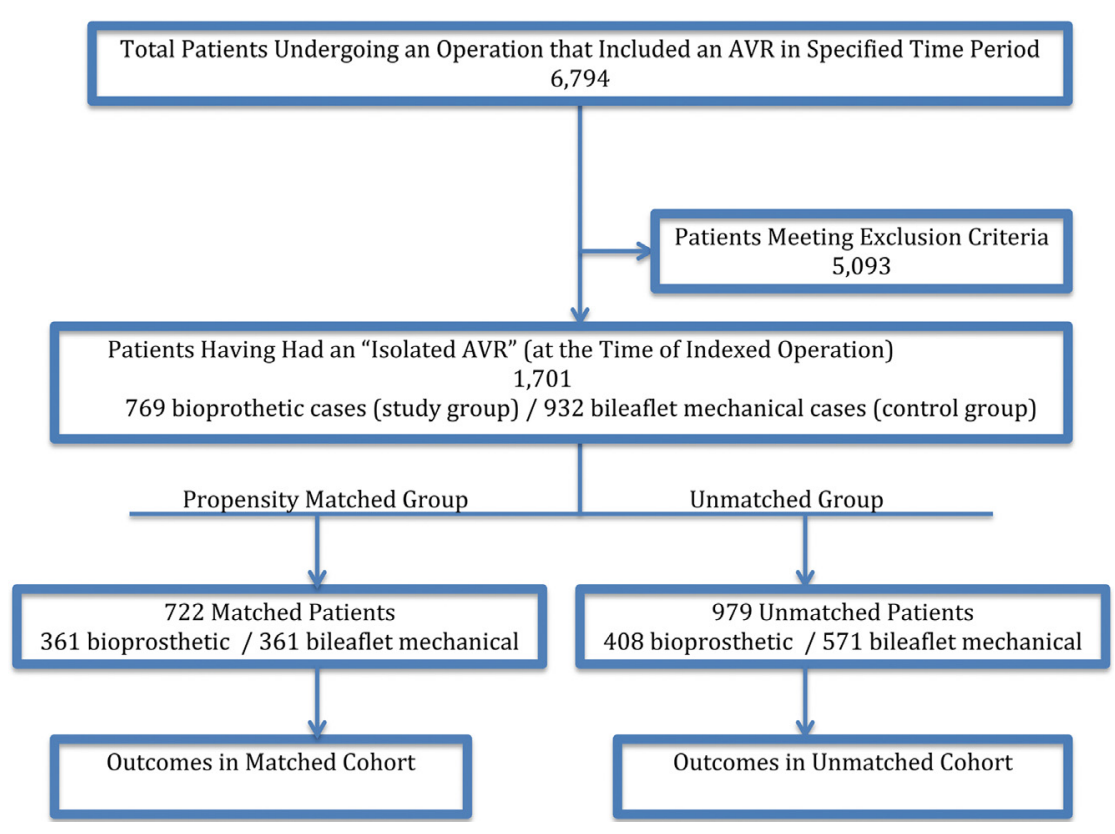

FIGURE 1. Flow chart for study design. AVR, Aortic valve replacement.

hypercholesterolemia. Patients receiving a mechanical prosthesis had more strokes, endocarditis, prior cardiac surgery, and a lower ejection fraction. To best replicate a clinical trial,

TABLE 1. Baseline characteristics for the entire cohort

\begin{tabular}{|c|c|c|c|}
\hline & \multicolumn{2}{|c|}{ Type of valve implanted } & \multirow[b]{2}{*}{$P$ value } \\
\hline & Bioprosthetic & Mechanical & \\
\hline Cases & 769 & 932 & \\
\hline \multicolumn{4}{|l|}{ Patient characteristic } \\
\hline Age (y) & $55.8 \pm 7.9$ & $51.1 \pm 9.7$ & $\leq .001$ \\
\hline Female & $28.2(217)$ & $28.6(267)$ & $\leq .871$ \\
\hline Body mass index & $28.6 \pm 5.8$ & $28.8 \pm 6.8$ & $\leq .653$ \\
\hline Hypertension & $51.8(398)$ & $41.1(383)$ & $\leq .001$ \\
\hline Hypercholesterolemia & $52.4(403)$ & $42.0(391)$ & $\leq .001$ \\
\hline Renal failure & $3.6(28)$ & $3.2(30)$ & $\leq .688$ \\
\hline Preoperative creatinine & $1.1 \pm 0.9$ & $1.1 \pm 0.8$ & $\leq .767$ \\
\hline Previous stroke & $2.7(21)$ & $5.0(46)$ & $\leq .024$ \\
\hline Congestive heart failure & $28.6(220)$ & $31.9(297)$ & $\leq .153$ \\
\hline Endocarditis & $5.3(41)$ & $8.4(78)$ & $\leq .017$ \\
\hline $\begin{array}{l}\text { New York Heart Association } \\
\text { functional class III-IV }\end{array}$ & $31.3(241)$ & $32.5(303)$ & $\leq .638$ \\
\hline Ejection fraction $(\%)$ & $60(55-65)$ & $60(50-63)$ & $\leq .001$ \\
\hline \multicolumn{4}{|l|}{ Operative characteristics } \\
\hline Previous cardiac surgery & $6.9(53)$ & $20.5(191)$ & $\leq .001$ \\
\hline $\begin{array}{l}\text { Previous coronary bypass } \\
\text { graft }\end{array}$ & $1.8(14)$ & $4.6(43)$ & $\leq .002$ \\
\hline Previous valve & $4.3(33)$ & $15.1(141)$ & $\leq .001$ \\
\hline $\begin{array}{l}\text { Cardiopulmonary bypass } \\
\text { time }(\mathrm{min})\end{array}$ & $111(85-154)$ & $128(97-180)$ & $\leq .001$ \\
\hline Ischemic time (min) & $80(61-114)$ & $90(69-125)$ & $\leq .001$ \\
\hline Concomitant aortic surgery & $21.6(166)$ & $32.9(307)$ & $\leq .001$ \\
\hline
\end{tabular}

we effectively matched 722 patients ( 361 stented bioprosthetic cases and 361 mechanical controls; 5413 patient-years of data; median follow-up 6.5 years; $42 \%$ of total cohort) using propensity scores. The predictive model from which matchedpairs were derived was robust, with an area under the receiver operator characteristic curve of 0.81 . Baseline characteristics for both the matched and unmatched cohorts are presented in Table 2. There were no significant differences for any of the measured covariates of interest in the matched cohort. The distributions for the valves implanted are listed in Table 3 . For the primary outcome of late survival follow-up was $99 \%$ complete (Table 4 ).

Secondary outcomes data was acquired on all patients as noted above. Questionnaire response rate was 74\% (1028 out of 1391). A responder bias analysis was performed to compare patient survey responders to nonresponders. ${ }^{13}$ Other than responders having a trend toward older age at the time of surgery ( $53 \pm 9$ years vs $52 \pm 9$ years for nonresponders; $P=.098$ ), no other discernible differences were noted. Gender mix; preoperative medical history; and laboratory data, incidence of stroke, complete heart block, cardiac arrest, and reoperations for bleeding during the postoperative period were similar between the 2 groups (all variables $P>.25$, data not shown).

\section{Short-Term Outcomes}

Mortality and morbidity were low for the duration of the study. Although there were many differences in short-term outcomes in the unmatched cohort, including 30-day mortality (Table 5), for the matched cohort short-term outcomes were very similar. Thirty-day mortality, postoperative 
TABLE 2. Baseline characteristics for the adjusted propensity-matched and unadjusted registry cohorts, by type of valve

\begin{tabular}{|c|c|c|c|c|c|c|}
\hline \multirow[b]{3}{*}{ Cases } & Bioprosthetic & Mechanical & \multirow{3}{*}{$P$ value } & Bioprosthetic & Mechanical & \multirow{3}{*}{$P$ value } \\
\hline & \multicolumn{2}{|c|}{ Propensity-matched cases } & & \multicolumn{2}{|c|}{ Registry cases } & \\
\hline & 361 & 361 & & 408 & 571 & \\
\hline \multicolumn{7}{|l|}{ Patient characteristic } \\
\hline Age & $53.9 \pm 9.0$ & $53.2 \pm 7.8$ & $\leq .298$ & $57.5 \pm 6.4$ & $49.7 \pm 10.5$ & $\leq .001$ \\
\hline Female & $30.2(109)$ & $29.6(107)$ & $\leq .935$ & $26.5(108)$ & $28.0(160)$ & $\leq .611$ \\
\hline Body mass index & $29.2(6.4)$ & $28.9(6.3)$ & $\leq .592$ & $28.1(5.2)$ & $28.7(7.2)$ & $\leq .213$ \\
\hline Hypertension & $52.1(188)$ & $46.8(169)$ & $\leq .180$ & $51.5(210)$ & $37.5(214)$ & $\leq .001$ \\
\hline Hypercholesterolemia & $47.1(170)$ & $50.1(181)$ & $\leq .457$ & $57.1(233)$ & $36.8(210)$ & $\leq .001$ \\
\hline Renal failure & 4.7 (17) & $3.0(11)$ & $\leq .335$ & $2.7(11)$ & $3.3(19)$ & $\leq .708$ \\
\hline Preoperative creatinine & $1.1(0.8)$ & $1.0(0.7)$ & $\leq .624$ & $1.0(0.9)$ & $1.1(0.9)$ & $\leq .482$ \\
\hline Previous stroke & $3.9(14)$ & $3.0(11)$ & $\leq .685$ & $1.7(7)$ & $6.2(35)$ & $\leq .001$ \\
\hline Congestive heart failure & $30.5(110)$ & $29.4(106)$ & $\leq .807$ & $27.0(110)$ & $33.5(191)$ & $\leq .035$ \\
\hline Endocarditis & $6.6(24)$ & $6.1(22)$ & $\leq .879$ & $4.2(17)$ & $9.8(56)$ & $\leq .001$ \\
\hline New York Heart Association functional class III-IV & $30.7(111)$ & $30.7(111)$ & $\leq 1.000$ & $31.9(130)$ & $33.6(192)$ & $\leq .638$ \\
\hline Ejection fraction $(\%)$ & $60(55-65)$ & $60(55-65)$ & $\leq .866$ & $60(55-65)$ & $55(50-65)$ & $\leq .001$ \\
\hline \multicolumn{7}{|l|}{ Operative characteristic } \\
\hline Previous cardiac surgery & $13.0(47)$ & $11.4(41)$ & $\leq .570$ & $1.5(6)$ & $26.4(151)$ & $\leq .001$ \\
\hline Previous coronary artery bypass graft & $3.0(11)$ & $3.6(13)$ & $\leq .836$ & $0.7(3)$ & $5.3(30)$ & $\leq .001$ \\
\hline Previous valve & $8.3(30)$ & $8.3(30)$ & $\leq 1.000$ & $0.7(3)$ & $19.4(111)$ & $\leq .001$ \\
\hline Cardiopulmonary bypass time (min) & $114(87-174)$ & $121(97-183)$ & $\leq .167$ & $110(81-145)$ & $132(120-247)$ & $\leq .001$ \\
\hline Ischemic time (min) & $80(61-129)$ & $87(68-135)$ & $\leq .100$ & $81(59-111)$ & $92(80-171)$ & $\leq .001$ \\
\hline Concomitant aortic surgery & $23.3(84)$ & $28.3(102)$ & $\leq .148$ & $20.1(82)$ & $35.9(205)$ & $\leq .001$ \\
\hline
\end{tabular}

Data are presented as mean \pm standard deviation, $\%(n)$, or median (interquartile range).

stroke, and re-exploration for bleeding showed no significant differences (Table 5). Length of hospital stay was the only short-term outcome to show a difference in the matched cohort, with patients receiving mechanical valves having a longer hospital stay $(P=.02)$ (Table 5), likely attributable to the time required to achieve therapeutic anticoagulation with warfarin therapy.

\section{Late Outcomes-Matched Cohort}

For late outcomes there was no identifiable difference in the primary outcome of survival out to 18 years of follow-up in the

TABLE 3. Distribution of valve types implanted

\begin{tabular}{lcc}
\hline \multicolumn{1}{c}{ Valve type } & Registry & Match \\
\hline Bioprosthetic & & \\
Total & 408 & 361 \\
Unknown & $2(0.5)$ & $1(0.3)$ \\
St Jude* Medical Biocor Porcine & $5(1.2)$ & $8(2.2)$ \\
Medtronic $\dagger$ Hancock II Porcine & $10(2.5)$ & $35(9.7)$ \\
Sorin $\ddagger$ Mitroflow Pericardial & $9(2.2)$ & $3(0.8)$ \\
Carpentier-Edwards $§$ Perimount Pericardial & $380(93.1)$ & $314(87.0)$ \\
Mechanical & & \\
Total & 571 & 361 \\
Unknown & $3(0.5)$ & $1(0.3)$ \\
Sorin $\ddagger$ Carbomedics Top Hat Bileaflet & $13(2.3)$ & $19(5.3)$ \\
On-X $\|$ Bileaflet & $5(0.9)$ & $23(6.4)$ \\
St Jude* Medical Bileaflet & $550(96.3)$ & $318(88.0)$ \\
\hline
\end{tabular}

Data are presented as n (\%). *St Jude Medical Inc, St Paul, Minn. †Medtronic, Minneapolis, Minn. †̦Sorin, Milan, Italy. §Edwards Lifesciences, Irvine, Calif. \|On-X Life Technologies, Austin, Tex. matched cohort (Figure 2). At 5, 10, 15, and 18 years, life table estimates for survival in the bioprosthetic group were $89 \% \pm$ $2 \%, 78 \% \pm 3 \%, 65 \% \pm 5 \%$, and $60 \% \pm 6 \%$, whereas for the mechanical group they were $88 \% \pm 2 \%, 79 \% \pm 3 \%$, $75 \% \pm 4 \%$, and $51 \% \pm 14 \%(P=.752)$. There were 65 deaths in the bioprosthetic group of which 16 were cardiacrelated. In the mechanical group there were 61 deaths of which 18 were cardiac-related. Still, with the cause of late death unknown for $43 \%$ of the cohort, the true influence of cardiac-related causes on late survival is most certainly higher for both groups (Table 6). Freedom from reoperation significantly favored mechanical prostheses $(P=.002)$, whereas freedom from major bleeding events significantly favored bioprosthetic valves $(P=.002)$ (Figures 3 and 4$)$. There was no difference in stroke between the 2 groups $(P=.33)$ (Figure 5).

TABLE 4. Late survival follow-up in years and patient-years

\begin{tabular}{ccccc}
\hline Cohort & $\begin{array}{c}\text { Median } \\
(\mathbf{I Q R})(\mathbf{y})\end{array}$ & Range (y) & Patient-years & $\begin{array}{c}\text { Vita status } \\
\text { follow-up (\%) }\end{array}$ \\
\hline Total cohort & & & & $99^{*}$ \\
Bioprosthetic & $5(3-9)$ & $1-18$ & 4998 & \\
Mechanical & $11(5-16)$ & $1-18$ & 9850 & \\
Matched cohort & & & & \\
Bioprosthetic & $7(4-11)$ & $1-18$ & 2738 & \\
Mechanical & $6(3-11)$ & $1-18$ & 2675 & \\
Registry cohort & & & & \\
Bioprosthetic & $5(3-8)$ & $1-18$ & 2260 & \\
Mechanical & $14(8-17)$ & $1-18$ & 7175 & \\
\hline
\end{tabular}

IQR, Interquartile range. $* 1682$ out of 1701 
TABLE 5. Short-term outcomes for both the adjusted propensity-matched and unadjusted registry cohorts

\begin{tabular}{|c|c|c|c|c|c|c|}
\hline \multirow[b]{3}{*}{ Patient characteristic } & \multicolumn{6}{|c|}{ Type of valve implanted } \\
\hline & Bioprosthetic & Mechanical & \multirow[b]{2}{*}{$P$ value } & Bioprosthetic & Mechanical & \multirow{2}{*}{$P$ value } \\
\hline & \multicolumn{2}{|c|}{ Propensity-matched cases } & & \multicolumn{2}{|c|}{ Registry cases } & \\
\hline Cases & 361 & 361 & & 408 & 571 & \\
\hline Reoperation for bleeding & $2.5(9)$ & $2.5(9)$ & $\leq 1.000$ & $1.5(6)$ & $3.3(19)$ & $\leq .098$ \\
\hline Postoperative stroke & $3.3(12)$ & $2.5(9)$ & $\leq .659$ & $1.5(6)$ & $4.6(26)$ & $\leq .010$ \\
\hline Heart block - PPM & $2.5(9)$ & $4.2(15)$ & $\leq .299$ & $0.7(3)$ & $5.1(29)$ & $\leq .001$ \\
\hline Cardiac arrest & $1.7(6)$ & $1.4(5)$ & $\leq 1.000$ & $1.2(5)$ & $2.3(13)$ & $\leq .335$ \\
\hline Ventilation $\mathrm{h}$ & $6(4-11)$ & $6(4-11)$ & $\leq .793$ & $6(4-10)$ & $7(4-13)$ & $\leq .004$ \\
\hline ICU h & $39(24-70)$ & $39(23-64)$ & $\leq .456$ & $38(24-62)$ & $39(24-70)$ & $\leq .700$ \\
\hline Hospital LOS (d) & $6(4-8)$ & $6(5-8)$ & $\leq .020$ & $5(5-7)$ & $7(5-9)$ & $\leq .001$ \\
\hline 30-d mortality & $1.9(7)$ & $1.4(5)$ & $\leq .773$ & $1.0(4)$ & $4.0(23)$ & $\leq .005$ \\
\hline
\end{tabular}

Data are presented as \% (n) or median (interquartile range). PPM, Permanent pacemaker; $I C U$, intensive care unit; $L O S$, length of stay.

\section{Late Outcomes-Registry Cohort}

The late outcomes for the unmatched cohort echoed those found in the matched cohort. There was no difference in late survival for the mechanical and bioprosthetic groups in the unmatched cohort, despite the fact that the mechanical group in the unmatched cohort had a greater preoperative risk profile than their bioprosthetic counterparts, leading to poorer short-term in-hospital outcomes. And although there was a trend toward increased stroke at late follow-up in the mechanical group from the unmatched cohort, this did not reach statistical significance $(P=.18)$. Freedom from reoperations significantly favored mechanical valves and freedom from major bleeding events favored bioprosthetic valves, just as it had in the matched cohort comparison (data not shown).

\section{DISCUSSION}

Improved outcomes in reoperative aortic valve surgery ${ }^{2,3}$ along with perceived improvements in the durability of second-generation bioprostheses ${ }^{4,5}$ have catalyzed a trend toward implanting bioprosthetic valves into younger patients. With the zealous embracing of percutaneous valvein-valve technology as a treatment alternative for SVD (a novel yet untested technology), this trend is certain to continue. ${ }^{6,14}$ Technologies to improve outcomes with mechanical valves have also materialized over time. Home monitoring systems to better regulate day-to-day international normalized ratio levels and mechanical valve prostheses touted to function at lower levels of anticoagulation have come to market. Still, the general trend continues to lean toward bioprosthetic valve implantation over mechanical valve implantation.

In our propensity-matched study comparing 361 patientpairs having undergone an AVR with a bileaflet mechanical valve or a bioprosthetic valve between 1992 and 2011, there was no difference in the primary outcome of late survival out to 18 years.

Our results parallel the results of a single randomized controlled trial comparing bileaflet mechanical valves to second-generation bioprostheses in middle-aged patients

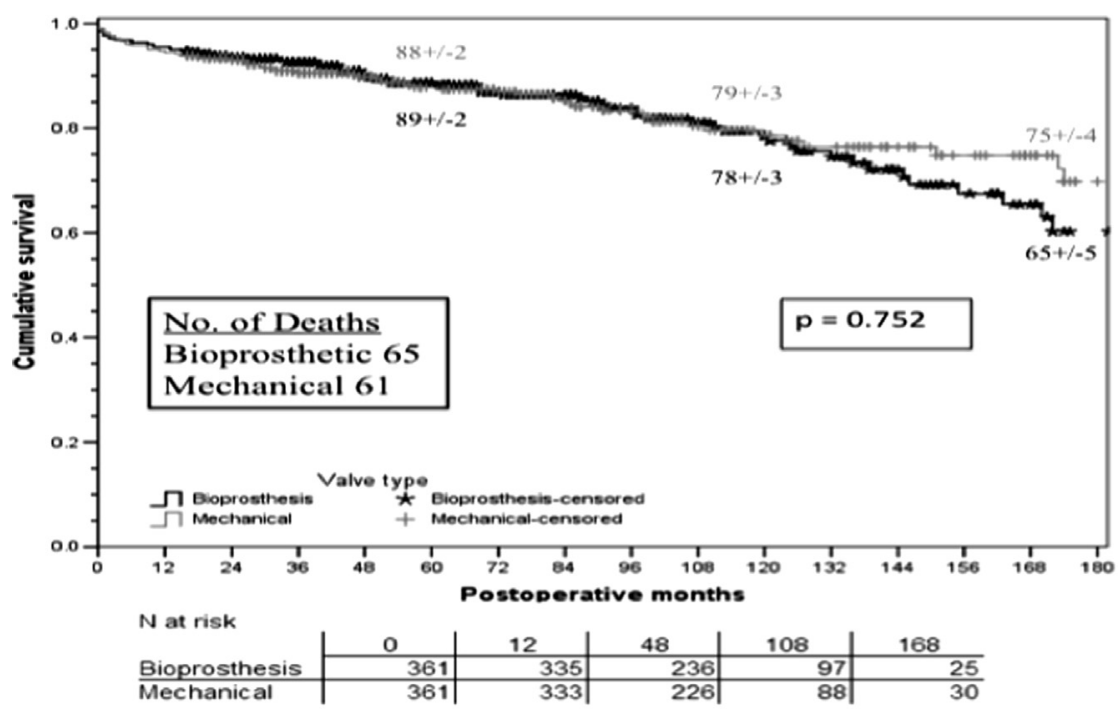

FIGURE 2. Kaplan-Meier survival curve comparing matched patient-pairs between bioprosthetic and mechanical valves. 
TABLE 6. Causes of late death

\begin{tabular}{lcc}
\hline \multicolumn{1}{c}{ Cause of death } & Bioprosthesis group & Mechanical group \\
\hline Cardiac arrest/myocardial & 12 & 13 \\
$\quad$ infarction & & \\
Endocarditis & 1 & 0 \\
Stroke & 3 & 5 \\
Cardiac-related & 16 & 18 \\
Pulmonary & 1 & 5 \\
Sepsis & 4 & 6 \\
MSOF & 3 & 2 \\
Renal failure & 1 & 1 \\
Cancer & 6 & 5 \\
Other (noncardiogenic) & 2 & 2 \\
Lost to follow-up & 32 & 22 \\
\hline
\end{tabular}

$M S O F$, Multisystem organ failure.

within the contemporary literature. ${ }^{12}$ In Stassano and colleagues" ${ }^{12}$ clinical trial of 155 randomized pairs, there was no difference in survival at a mean 8.8 years followed out to 13.4 years. On the contrary, Brown and colleagues ${ }^{9}$ reported a survival advantage for mechanical valves over bioprostheses in an observational study of 220 casematched pairs at 10 years' follow-up. Stassano and colleagues $^{12}$ and Brown and colleagues ${ }^{9}$ each assessed middle-aged patients with an age range of 50 to 70 years, whereas our study only included patients younger than age 65 years. Notably, at a mean age of 53.5 years, our matched cohort was approximately 10 years younger than the mean age for patients in either of these 2 studies. Other differences in study design are also important when interpreting the results. We chose to include only those patients undergoing an isolated AVR. Focused solely on isolated AVRs, we derived a matched group of 361 patientpairs with statistically similar postoperative complications, including 30-day mortality. This suggests evenly matched patient-pairs capable of producing credible late outcomes data for insightful interpretation. Stassano and colleagues ${ }^{12}$ and Brown and colleagues ${ }^{9}$ included patients having had either an AVR or an AVR plus coronary artery bypass grafting procedure. Although this is of no consequence for Stassano and colleagues, ${ }^{12}$ who performed a randomized trial, it could certainly enable confounding and disproportionate myocardial ischemic burdens to have materialized within the presumed evenly matched patients of the analysis by Brown and colleagues. ${ }^{9}$ This could have contributed to the difference in long-term mortality seen within that study despite efforts to control for this through statistical measures. The significant difference in 30-day mortality noted in the matched pairs of the analysis by Brown and colleagues ${ }^{9}$ (with the bioprosthetic group having had a higher mortality), fuels this speculation. If 2 evenly matched groups differ only by valve implant, theoretically no difference in 30-day mortality should arise.

More recently, 2 additional observational studies have also suggested a survival benefit of mechanical valves at late follow-up. Badhwar and colleagues ${ }^{10}$ performed a propensity-matched analysis comparing secondgeneration bioprostheses to the On-X bileaflet mechanical valve (On-X Life Technologies, Austin, Tex) in a very small cohort of patients (142 total patients) younger than age 65

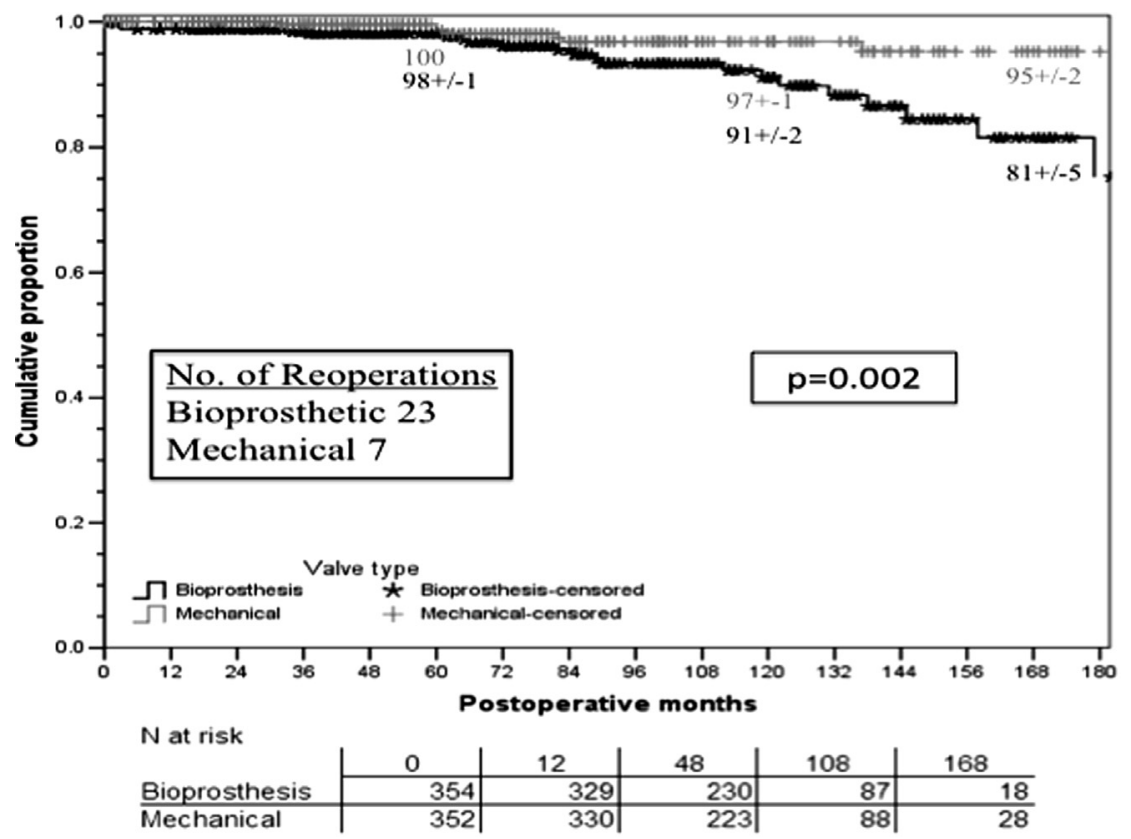

FIGURE 3. Kaplan-Meier curve comparing freedom from reoperations between matched patient-pairs with bioprosthetic and mechanical valves. 


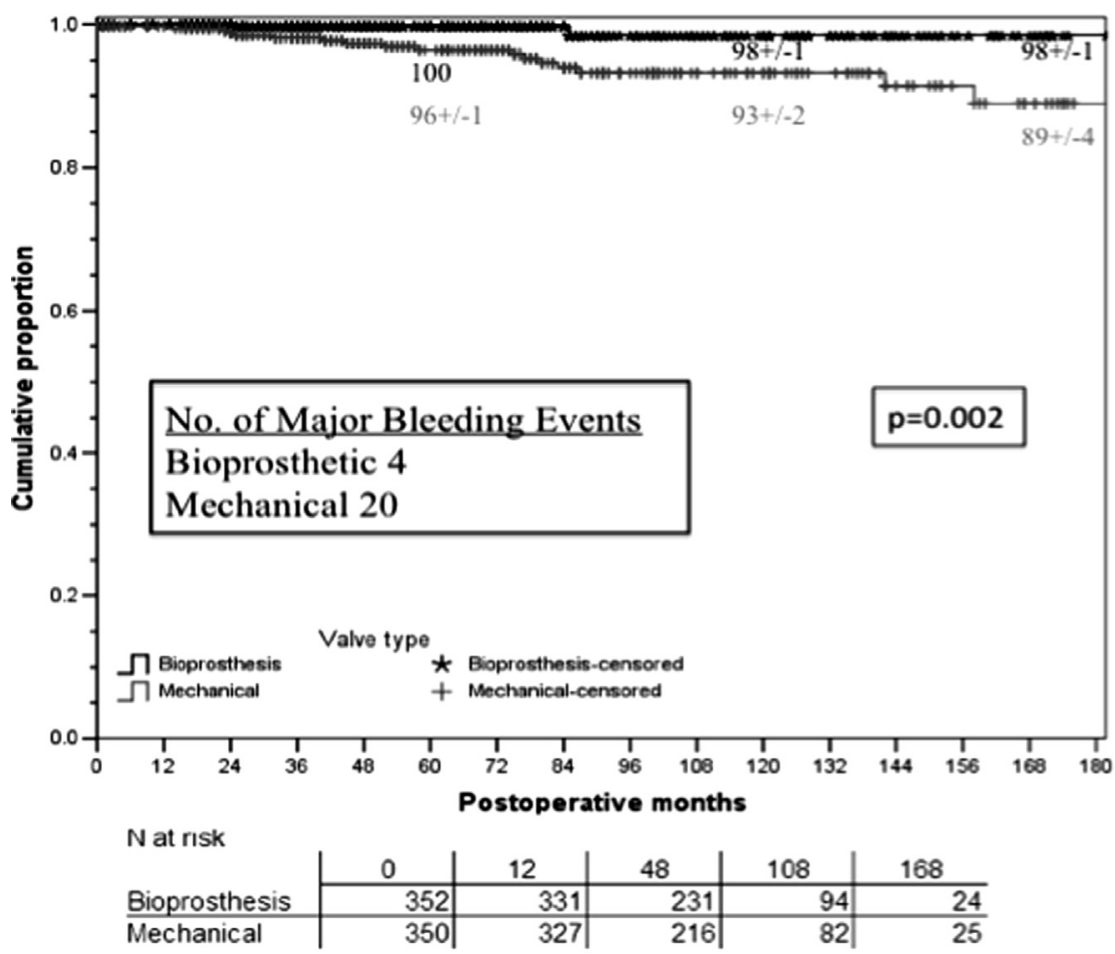

FIGURE 4. Kaplan-Meier curve comparing major bleeding events between matched patient-pairs with bioprosthetic and mechanical valves.

years with a modest median follow-up of 4 years. Interestingly, the study included all primary isolated left-sided heart valve replacements (mitral and aortic). Despite propensity matching, $52 \%$ of patients in the mechanical group underwent a mitral valve replacement $(48 \%$ underwent an AVR) and were compared with a presumably evenly matched bioprosthetic group where only $15 \%$ underwent a mitral valve replacement ( $85 \%$ underwent an AVR). A

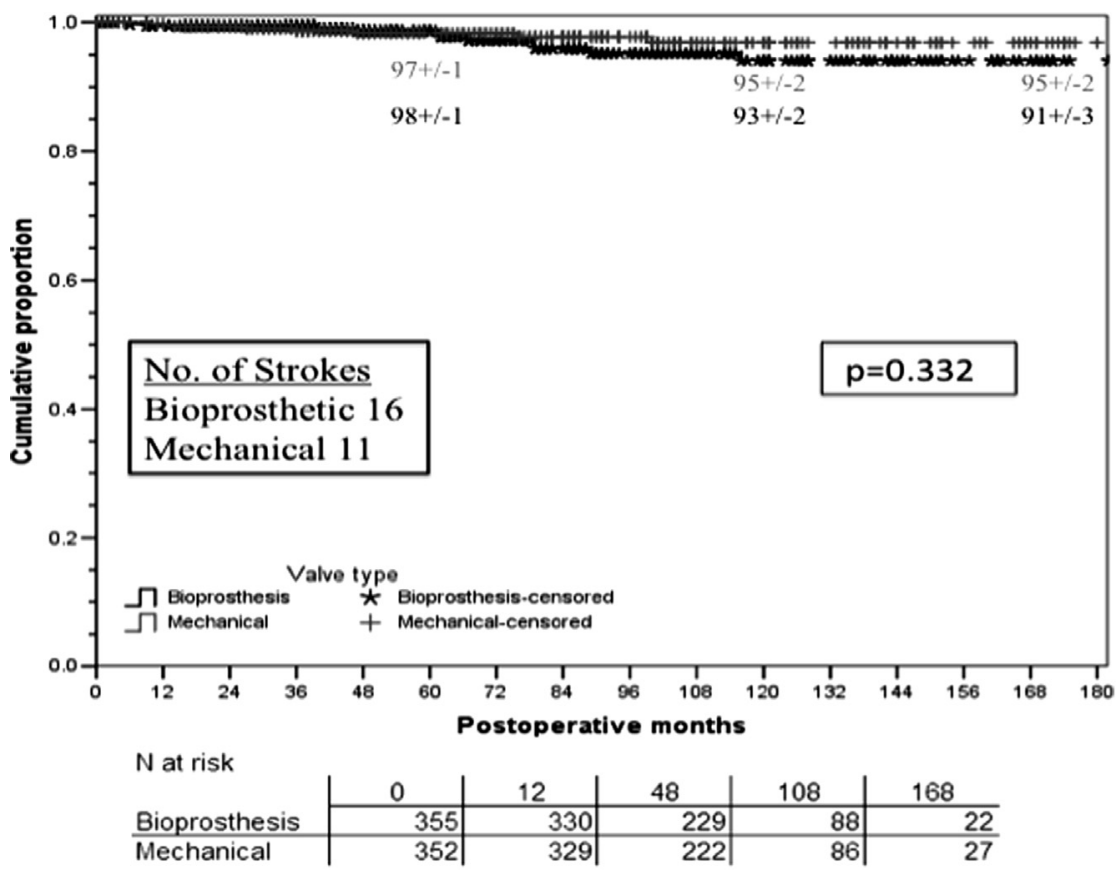

FIGURE 5. Kaplan-Meier curve comparing stroke rates between matched patient-pairs with bioprosthetic and mechanical valves. 
survival advantage was noted for the On-X mechanical valves at 7.5 years' follow-up. However, to be confident that patients have been evenly matched when there is a significantly disproportionate amount of structural left heart disease in the 2 comparative groups, regardless of the statistical measures, is difficult. The opportunity for confounding is substantial.

Weber and colleagues ${ }^{11}$ performed a thorough propensity-matched analysis to derive 103 patient-pairs with mean age 50 years but with a very short mean follow-up of only 2.75 years. Although Weber and colleagues ${ }^{11}$ also had inclusion criteria enabling concomitant coronary revascularization or additional valve repairs to have occurred, there was no difference in early mortality amongst the 2 matched groups. A crude mortality survival advantage was noted for mechanical valves over biologic valves in their propensity-matched group. A review of their article showed that after adjusting for 13 additional potential confounding variables, this survival advantage was no longer present $(P=.203)$. Despite this, the authors still concluded that patients aged younger than 60 years incur a survival advantage with mechanical valves.

Somewhat surprisingly, in our unmatched cohort we were again unable to detect a difference in late survival between patients receiving mechanical versus bioprosthetic valves. Although the mechanical group in the unmatched cohort had a higher risk profile (ie, more redo operations, endocarditis, prior strokes, and poorer ejection fraction), the bioprosthetic group was significantly older with a mean age of 57.5 versus 49.7 years, which may have been the equalizing factor to mitigate any survival differences.

The results for secondary outcomes in our study, with major bleeding occurring more often with mechanical valves, reoperations occurring more often with bioprosthetic valves, and no difference in stroke rates, are similar to previous reports. Second-generation bioprostheses are more durable than first-generation bioprostheses and anticoagulation is likely better monitored for mechanical valves in the current era than prior. However, the overall result relative to 1 another remains the same: bioprosthetic valves result in more reoperations and mechanical valves result in more bleeding events.

Our study has several strengths. With 361 evenly matched pairs, this is the largest cohort comparing bioprosthetic and bileaflet mechanical valves in the literature. By focusing on isolated AVR and excluding concomitant coronary revascularization and additional valve replacement procedures, key confounders were eliminated from the analysis. Our propensity model was robust and our follow-up for the primary outcome was $99 \%$ complete over an extended period.

\section{Limitations}

Ours was a retrospective, single-center observational study. All such studies are at risk for an underestimation of events. Despite a systematic and exhaustive chart review with multiple layers of cross-referencing the database to ensure accuracy in addition to input from patient questionnaires, an underestimation of late events (eg, reoperations, major bleeding, and strokes) is still plausible. Specific to the questionnaire responses, recall bias, where patients simply forget prior adverse events (having happened several years prior), will result in some degree of underestimation. Additionally, it is possible that those who did not respond to our questionnaire (for whom secondary outcome data was acquired solely from the EMR), did in fact have worse secondary outcomes than responders and thus the overall representation is underestimated. Our responder bias analysis suggests otherwise, because no clear differences were identified; this supports that their information is missing at random but this cannot be known with absolute certainty. Assuming such underestimation is evenly distributed across the cohort, the overall effect of such underestimation on group comparisons between patients with a bioprosthesis and a mechanical prosthesis that have been evenly matched in a pairwise fashion should be rather noncontributory. ${ }^{13}$

Despite our efforts to create a clinically matched subset of comparable patients using a comprehensive stepwise logistic regression propensity-score analysis, there is always the potential for confounding due to unforeseen and unmeasurable covariates.

\section{CONCLUSIONS}

In nonelderly (ie, younger than age 65 years) patient populations, despite an increase in bleeding events with mechanical valves and an increased rate of reoperation with stented bioprosthetic valves, choice of valve prosthesis does not appear to affect late survival.

\section{References}

1. Bonow RO, Carabello BA, Chatterjee K, de Leon AC Jr, Faxon DP, Freed MD, et al. 2008 focused update incorporated into the ACC/AHA 2006 guidelines for the management of patients with valvular heart disease: a report of the American College of Cardiology/American Heart Association Task Force on Practice Guidelines. J Am Coll Cardiol. 2008;52:e1-142.

2. Leontyev S, Borger MA, Davierwala P, Walther T, Lehmann S, Kempfert J, et al. Redo aortic valve surgery: early and late outcomes. Ann Thorac Surg. 2011;91: 1120-6.

3. Ghanta RK, Kaneko T, Gammie JS, Sheng S, Aranki SF. Evolving trends of reoperative coronary artery bypass grafting: an analysis of the Society of Thoracic Surgeons adult cardiac surgery database. J Thorac Cardiovasc Surg. 2013;145: 364-72.

4. David TE, Armstrong S, Maganti M. Hancock II bioprosthesis for aortic valve replacement: the gold standard of bioprosthetic valve durability? Ann Thorac Surg. 2010;90:775-81

5. McClure RS, Narayanasamy N, Wiegerinck E, Lipsitz S, Maloney A, Byrne JG, et al. Late outcomes for aortic valve replacement with the Carpentier-Edwards pericardial bioprosthesis: up to 17-year follow-up in 1,000 patients. J Thorac Cardiovasc Surg. 2010;89:1410-6.

6. Ye J, Webb JG, Cheung A, Soon JL, Wood D, Thompson CR, et al. Transapical transcatheter aortic valve-in-valve implantation: clinical and hemodynamic outcomes beyond 2 years. J Thorac Cardiovasc Surg. 2013;145:1554-62.

7. Brown JM, O'Brien SM, Wu C, Sikora JH, Griffith BP, Gammie JS. Isolated aortic valve replacement in North America comprising 108,687 patients in 10 
years: changes in risks, valve types, and outcomes in the Society of Thoracic Surgeons national database. J Thorac Cardiovasc Surg. 2009;137:82-90.

8. Dunning J, Gao H, Chambers J, Moat N, Murphy G, Pagano D, et al. Aortic valve surgery: marked increases in volume and significant decreases in mechanical valve use-an analysis of 41,227 patients over 5 years from the Society for Cardiothoracic Surgery in Great Britain and Ireland National database. J Thorac Cardiovasc Surg. 2011;142:776-82.

9. Brown ML, Schaff HV, Lahr BD, Mullany CJ, Sundt TM, Dearani JA, et al. Aortic valve replacement in patients aged 50 to 70 years: improved outcome with mechanical versus biologic prostheses. J Thorac Cardiovasc Surg. 2008;135:878-84.

10. Badhwar V, Ofenloch JC, Rovin JD, van Gelder HM, Jacobs JP. Noninferiority of closely monitored mechanical valves to bioprostheses overshadowed by early mortality benefit in younger patients. Ann Thorac Surg. 2012;93:748-53.
11. Weber A, Noureddine H, Englberger L, Dick F, Gahl B, Aymard T, et al. Ten-year comparison of pericardial tissue valves versus mechanical prostheses for aortic valve replacement in patients younger than 60 years of age. J Thorac Cardiovasc Surg. 2012;144:1075-83.

12. Stassano P, Di Tommaso L, Monaco M, Iorio F, Pepino P, Spampinato N, et al. Aortic valve replacement: a prospective randomized evaluation of mechanical versus biological valves in patients ages 55 to 70 years. J Am Coll Cardiol. 2009;54:1862-8.

13. Johnson TP, Wislar JS. Response rates and nonresponse errors in surveys. JAMA 2012;307:1805-6.

14. Dvir D, Webb JG, Bleiziffer S. Transcatheter aortic valve replacement for degenerative bioprosthetic surgical valves: results from the global valve-in-valve registry. Circulation. 2012;126:2335-44. 